\title{
Efecto de especies forrajeras sobre las bases intercambiables de un suelo sulfatado ácido del municipio de Paipa (Colombia)
}

\section{The effect of the fodder species over the interchangeable bases of sulfated acid soil in municipality of Paipa (Colombia)}

Efeito de espécies forrageiras em bases trocáveis de um solo ácido sulfatada Township Paipa (Colômbia)

\author{
Andrea Angélica Bernal Figueroa ${ }^{1}$ \& Fabio Emilio Forero Ulloa ${ }^{2}$ \\ ${ }^{1}$ Bióloga, Candidata a Magister en Ingeniería Ambiental. ${ }^{2}$ Ingeniero Agrónomo, \\ Especialista en Frutales de Clima Frío, Especialista en Ingeniería Ambiental. \\ MSc en Ciencias Agrarias con énfasis en suelos y aguas \\ 1,2 Universidad Pedagógica y Tecnológica de Colombia, Sede Central. Tunja. Boyacá. Colombia. \\ 1anbernal@gmail.com, 2guatoquero@gmail.com
}

\section{Resumen}

Una de las limitaciones en suelos sulfatados ácidos (SSA) está relacionada con la inhibición en la absorción de bases intercambiables generada principalmente por excesos de aluminio de cambio, esto afecta su productividad y limita la disponibilidad de nutrientes para las plantas. La presente investigación evaluó en invernadero el efecto de remolacha forrajera (Beta vulgaris L.), nabo forrajero (Brassica rapa L.) y rábano forrajero (Raphanus sativus L.) sobre las bases intercambiables de un SSA, contrastado con un suelo no sulfatado, con el fin de identificar especies para manejo y rehabilitación de estos suelos en Paipa (Boyacá). Se implementó un diseño experimental con 6 tratamientos y 3 repeticiones en los cuales se realizó la medición de bases en el suelo antes y después de la siembra de las especies, y se midió área foliar y altura de las plantas al finalizar su periodo vegetativo. Se presentaron diferencias estadísticas significativas $(P<0,01)$ en la concentración de bases, así como en las variables área foliar y altura de la planta. Los resultados indicaron que el crecimiento del nabo (B. rapa) y rábano
(R. sativus) forrajeros, junto con el uso complementario de enmiendas calcáreas como correctivo, indujeron a un aumento en las concentraciones de $\mathrm{Ca}, \mathrm{Mg}, \mathrm{K}$ y $\mathrm{Na}$ en el SSA, y presentaron un mejor crecimiento y resistencia a los mismos. Igualmente, la siembra de remolacha forrajera $(B$. vulgaris) generó efectos positivos en el suelo; sin embargo, la altura y área foliar de esta especie se afectaron en el SSA, lo cual limita su productividad.

Palabras clave: manejo, nabo forrajero, rábano forrajero, rehabilitación, remolacha forrajera.

\section{Abstract \\ One of the limitations on acid sulphated soils (SSA) is related to the inhibition in the absorption of in- terchangeable bases generated mainly by exces- ses of aluminum oxide of change; this affects its productivity and limits the availability of nutrients for plants. This research evaluated in greenhou- se, the effect of fodder beet (Beta vulgaris L.), forage turnip (Brassica rapa L.) and fodder radish (Raphanus sativus L.) on the interchangeable}


bases of an SSA, contrasted with a not sulfated ground, in order to identify species for management and rehabilitation of these soils in Paipa (Boyacá). An experimental design was implemented with 6 treatments and 3 repetitions in which the basis measurement was made in the ground before and after the species plating and the measured leaf area and plants height when their vegetative period ended. Several significative statistic differences were found $(P<0.01)$ in the basis concentration, as well as in the variables in the leaf area and height of the plant. The results indicated that the growth of the turnip (B. rapa) and horseradish ( $R$. sativus) fodder, along with the complementary use of calcareous amendments as a corrective, led to an increase in the concentrations of $\mathrm{Ca}$, $\mathrm{Mg}, \mathrm{K}$, and $\mathrm{Na}$ in the SSA, and presented a better growth and resistance to them. Similarly, the planting of fodder beet (B. vulgaris) generated positive effects in the soil; however, the height and leaf area of this species were affected in the SSA, which limits their productivity.

Key-words: management, forage turnip, fodder radish, rehabilitation, fodder beet

\section{Resumo:}

Uma limitação em solos sulfatados ácidos (SSA) está relacionada com a inibição na absorção das bases trocáveis, gerada principalmente por excesso de alumínio trocável, isso afeta a produtividade e limita a disponibilidade de nutrientes para as plantas. Esta pesquisa avaliou em casa de vegetação o efeito da beterraba forrageira (Beta vulgaris L.), os nabos forrageiros (Brassica rapa L.) e nabo forrageiro (Raphanus sativus L.) sobre bases trocáveis em um SSA contrastado com solo não-sulfatado, a fim de identificar as espécies de gestão e reabilitação destes solos em Paipa (Boyacá). Foi implementado um delineamento experimental com 6 tratamentos e três repetições, com medição de bases no solo antes e após o plantio de espécies, e área foliar e altura das plantas foi medida no final do seu período de crescimento. Apresentaram-se diferenças estatisticamente significativas $(P<0,01)$ na concentração de bases, bem como nas variáveis área folhar e altura de planta. Os resultados indicaram que o crescimento do nabo (B. rapa) e rábano forrageiro (R.sativus), juntamente com a utilização complementar de calagem como corretivo, levou a um aumento das concentrações de $\mathrm{Ca}, \mathrm{Mg}, \mathrm{Ke}$ $\mathrm{Na}$ no SSA, e apresentou melhor crescimento e resistência a eles. Da mesma forma, o plantio de beterraba forrageira (B. vulgaris) produziu efeitos positivos sobre o solo; no entanto, altura e área foliar desta espécie foram afetadas no SSA, o que limita sua produtividade.

Palavras-chave: manejo, nabo forrageiro, rábano forrageiro, reabilitação, beterraba forrageira

\section{Introducción}

Son múltiples las causas de la acidez del suelo, entre algunas se señalan su origen por materiales parentales ácidos, alta percolación por excesiva pluviosidad, uso prolongado e histórico de fertilizantes con residuos ácidos (nitrogenados, ureicos y amoniacales) o la composición de la materia orgánica, entre otros; estos procesos contribuyen junto a otros factores a reducir rendimientos y producción (Ocampo, Chavarriaga \& Ceballos, 2007; Osorno, 2012).
Específicamente, suelo sulfatado ácido (SSA) es el nombre dado a los suelos que contienen sulfuro de hierro, principalmente pirita férrico mineral, o que contienen ácidos producto de la oxidación de sulfuros (National Working Party on Acid Sulfate Soils, 2000). Son característicos de las zonas bajas que se inundan con frecuencia o en su efecto permanecen gran parte del tiempo inundados, también se encuentran en los deltas y estuarios de los ríos, lo cual genera ácidos 
solubles (ácido sulfúrico) producto de mezclas de sedimentos orgánicos y/o minerales ricos en hierro y azufre (Cabrales, 2007; Castro \& Munevar, 2011; Montaño \& Forero, 2013). Cuando los SSA se drenan, los sulfuros se oxidan y se forman horizontes sulfúricos con $\mathrm{pH}$ extremadamente ácidos $(<4)$ con altas concentraciones de azufre $(>100 \mathrm{ppm})$ y aluminio intercambiable (>5 $\mathrm{cmol}_{\mathrm{c}} / \mathrm{kg}$ ) (Dent \& Dawson, 2000; Castro \& Munevar, 2011).

A nivel mundial se estima que el área afectada por estos suelos es de 24 millones de ha (Dent \& Dawson, 2000; Combatt, Palencia \& Marín, 2003; Cabrales, 2007). En Colombia cerca del $85 \%$ de los suelos son ácidos (Casierra \& Aguilar, 2007) y en el departamento de Boyacá se encuentran alrededor de 3.000 ha de SSA, en el Distrito de Riego del Alto Chicamocha - DRACH (Hernández \& Viteri, 2006). El desconocimiento de la problemática por estos suelos, ha generado el uso incorrecto de los mismos, lo que incrementa los problemas ambientales asociados, repercute en el desarrollo sostenible de los ecosistemas conexos y, a su vez, genera la degradación de los recursos suelo y agua con importantes implicaciones ambientales y económicas (Combatt, Martínez, Cabrales, Martínez, Castillo \& Palencia, 2005).

La toxicidad generada en estos suelos, principalmente por el aluminio intercambiable y en muchos casos por ácido sulfúrico y hierro, alteran el equilibrio iónico del mismo, inhiben la absorción de $\mathrm{Ca}^{+2}, \mathrm{Mg}^{+2}$ y $\mathrm{P}$ y afectan directamente su fertilidad (Castro \& Munevar, 2011). Muchas especies vegetales tienen mecanismos eficientes para la exudación de iones a través de ácidos orgánicos por las raíces; asimismo, la proliferación de determinadas rizobacterias y micorrizas estimulan el crecimiento de las plantas bajo condiciones ambientales estresantes (Taylor et al., 2000). En este contexto, la presente investigación evaluó el efecto de remolacha forrajera ( $B$. vulgaris), nabo forrajero (B. rapa) y rábano forrajero ( $R$. sativus) sobre las bases intercambiables ( $\mathrm{Ca}, \mathrm{Mg}, \mathrm{K}$ y Na) de un SSA, contrastado con un suelo no sulfatado, a nivel de invernadero, como posibles especies vegetales para el manejo y rehabilitación de estos suelos en el municipio de Paipa (Boyacá).

\section{Materiales y métodos}

EI SSA se colectó en un lote del sector Varguitas del Municipio de Paipa (Boyacá, Colombia) a una altura promedio de $2.500 \mathrm{msnm}$, con coordenadas $5^{\circ}$ 4' $51^{\prime \prime} \mathrm{N}$ y $73^{\circ} 3^{\prime \prime}$ 29' W. Se tomó una muestra de $1 \mathrm{~kg}$ de suelo según la metodología planteada por Bautista, Delfín \& Palacio (2011) para realizar la medición inicial de los parámetros a evaluar; de igual forma, se recolectaron $135 \mathrm{~kg}$ de SSA a una profundidad no superior a $20 \mathrm{~cm}$ y se trasladaron al Jardín Botánico de la Universidad Pedagógica y Tecnológica de Colombia - UPTC (Tunja) para el montaje de las unidades experimentales (UE).

Se utilizó un diseño experimental con 6 tratamientos (Tabla 1), 3 repeticiones y dos tipos de suelo; cada UE estuvo compuesta por tres bolsas de polietileno para vivero con $5 \mathrm{~kg}$ de suelo, para un total de 54 UE. De acuerdo con los resultados arrojados por la medición cero (0) de los dos tipos de suelo, se realizaron los cálculos de las cantidades necesarias de enmienda a emplear; así, en el SSA se agregaron $20 \mathrm{t} \mathrm{ha}^{-1}$ de cal dolomita y en el suelo no sulfatado se adicionaron $8.8 \mathrm{t} \mathrm{ha}^{-1}$ de cal agrícola. Se realizó el montaje de las UE y luego de un periodo de reacción de la enmienda de 60 días, se llevó a cabo la siembra de 4 semillas por bolsa. 
Tabla 1. Descripción de los tratamientos

Tratamiento

[T1]

[T2]

\section{Descripción}

Suelo no sulfatado + Remolacha forrajera (Beta vulgaris L.)

Suelo no sulfatado + Nabo forrajero (Brassica rapa L.)

Suelo no sulfatado + Rábano forrajero (Raphanus sativus L.)

Suelo sulfatado ácido + Remolacha forrajera (Beta vulgaris L.)

Suelo sulfatado ácido + Nabo forrajero + (Brassica rapa L.)

Suelo sulfatado ácido + Rábano forrajero (Raphanus sativus L.)
Para la evaluación de las variables respuesta, se llevó a cabo la medición de $\mathrm{Ca}, \mathrm{Mg}, \mathrm{K}$ y $\mathrm{Na}$ en el suelo, mediante el método de acetato de amonio $1 \mathrm{~N}$ y neutro, de acuerdo con el IGAC (2006), en el Laboratorio de Docencia de Suelos de la Facultad de Ciencias Agropecuarias de la UPTC. Se realizó una medición 0 (cero) correspondiente al suelo sin corrección agronómica, una medición inicial luego del periodo de reacción de la enmienda y una medición final después de la cosecha de las especies (120 días después de la siembra). Al finalizar el periodo vegetativo se determinó la respuesta agronómica de las plantas en cada tratamiento; el área foliar se calculó con el equipo $\mathrm{Cl}-202$ Leaf Area Meter, y la altura de las plantas se midió desde el cuello de la raíz hasta la hoja superior con flexómetro. Para el procesamiento de los datos se realizó un análisis de varianza (ANOVA) para determinar diferencias estadísticas entre tratamientos, y se utilizó la prueba de comparación de promedios Tukey con una confiabilidad del $95 \%$ con el programa estadístico SAS v. 9.1 (Cary, N.C). Las comparaciones se llevaron a cabo entre el suelo después del proceso de encalamiento (inicial) y luego de la cosecha de las plantas (final).

\section{Resultados}

Se presentaron diferencias estadísticamente significativas $(P<0,01)$ en las concentraciones de $\mathrm{Ca}, \mathrm{Mg}, \mathrm{K}$ y $\mathrm{Na}$ en la medición final, en comparación a la inicial (después del encalado) (Figura 1 A, B, C y D). En los niveles de Ca se observó incremento de forma equivalente en la medición final, tanto en los tratamientos correspondientes al SSA como en los del suelo no sulfatado (Figura 1A); mientras que el Mg aumentó considerablemente en el SSA a valores similares con las tres especies (Figura 1B). El contenido de $\mathrm{K}$ disminuyó en el suelo no sulfatado, donde el nivel más bajo se registró con la siembra de remolacha (B. vulgaris); sin embargo, estos valores son notablemente superiores a los encontrados en el SSA, tanto antes como después de la siembra (Figura 1C). Con respecto a la concentración de $\mathrm{Na}$, se registró incremento en la totalidad de tratamientos; en el suelo no sulfatado el mayor valor se evidenció con la siembra de nabo (B. rapa), mientras que en el SSA aumentó de forma similar con la siembra de las tres especies (Figura 1D). 


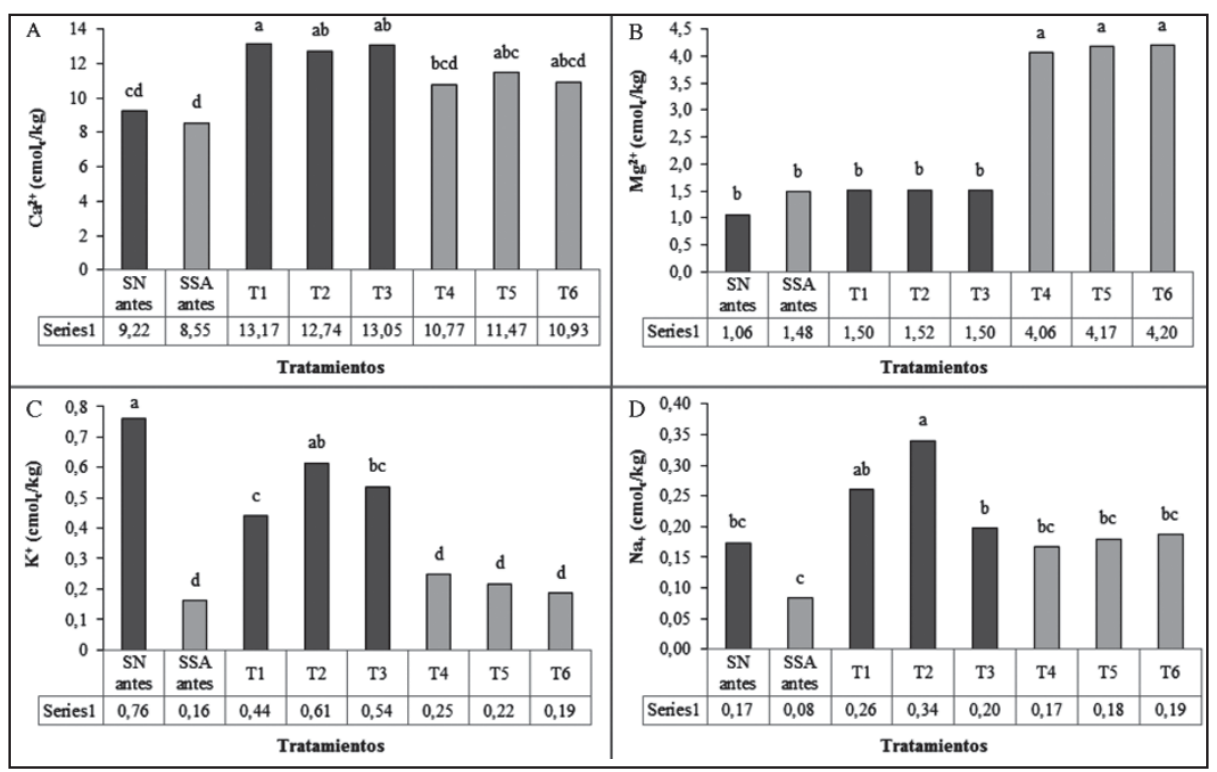

Figura 1. Efecto de especies vegetales sobre: A. Calcio, B. Magnesio, C. Potasio y D. Sodio de un suelo sulfatado ácido (SSA) y un suelo no sulfatado (SN). SN antes: medición antes de la siembra; SSA antes: medición antes de la siembra. Promedios seguidos de letras distintas en la misma serie, presentan diferencias significativas según la prueba de Tukey (5\%).

En cuanto a la respuesta fisiológica de las plantas, las variables área foliar y altura de la planta presentaron diferencias estadísticamente significativas $(P<0,01)$. La remolacha $(B$. vulgaris $)$ presentó mayor área foliar en el suelo no sulfatado, mientras que el nabo (B. rapa) obtuvo un valor levemente mayor en el SSA. En cuanto a la altura de las plantas, aunque estadísticamente las especies se comportaron de forma similar en los dos tipos de suelo, es importante resaltar que se notó una disminución de esta variable en la remolacha ( $B$. vulgaris) y el rábano ( $R$. sativus) sembrados en el SSA. Por su parte, el nabo $(B$. rapa), al igual que en área foliar, presentó una mayor altura en el SSA (Figura 2A y B).
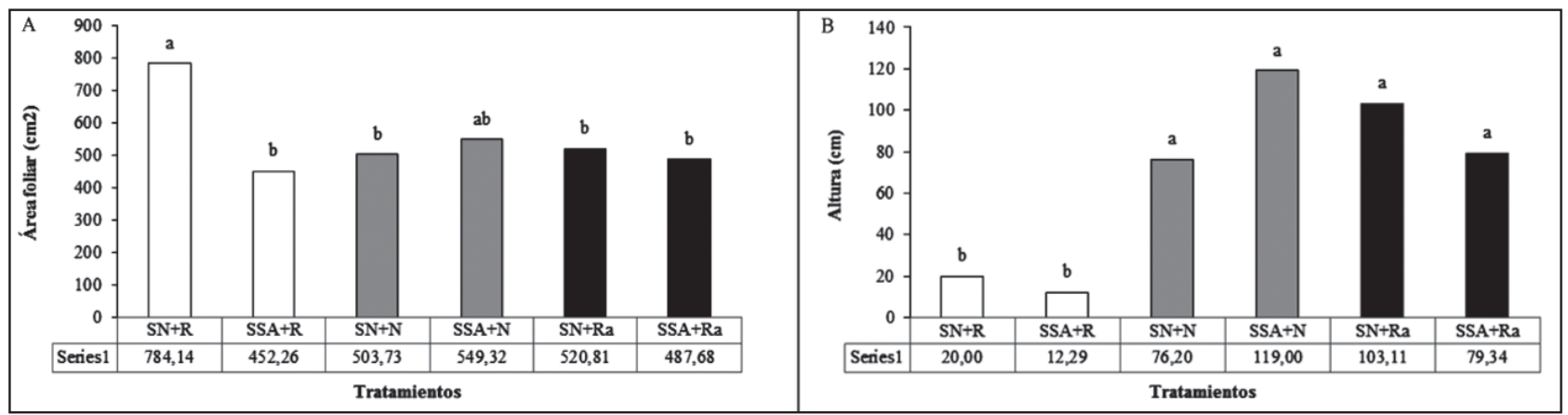

Figura 2. Medición de variables agronómicas: A. Área foliar y B. Altura de las plantas en un suelo sulfatado ácido (SSA) y un suelo no sulfatado (SN). R: Remolacha forrajera; N: Nabo forrajero;

Ra: Rábano forrajero. Promedios seguidos de letras distintas en la misma serie, presentan diferencias significativas según la prueba de Tukey (5\%). 


\section{Discusión}

De acuerdo con Osorno (2012) y Castro \& Munevar (2011), el mejoramiento que se evidenció en las concentraciones de las bases intercambiables, tanto en el suelo no sulfatado como en el SSA, se presentó a causa de la aplicación de cal, debido a que la acción fundamental de esta técnica es el mejoramiento o la corrección de las condiciones químicas del suelo, lo que a su vez se vio favorecido por la incorporación de dichos materiales encalantes unos meses antes de la siembra del cultivo, lo cual propicia un mejor ambiente para el crecimiento y desarrollo radical de las plantas. De igual forma, pudo contribuir con el mejoramiento de las bases, tanto la absorción de las plantas, como su interacción con los componentes del suelo (Dakora \& Phillips (2002); Hinsinger, Plassard, Tang \& Jaillard, 2003).

Al respecto, Lazcano (2001) manifiesta que en aquellos suelos donde la acidez limita el rendimiento del cultivo, la aplicación de cal agrícola es la mejor práctica de manejo e influye en la disponibilidad de nutrientes para la planta; igualmente, Molina (2008) indica que el encalado, junto con la siembra de especies tolerantes, constituyen las prácticas más apropiadas y económicas para corregir los problemas de acidez, lo cual a su vez permite mejorar la conservación del suelo y el paisaje al establecer una cobertura vegetal. Castro \& Munevar (2011) y Osorno (2012), por su parte, señalan que las prácticas de manejo que conllevan al mejoramiento químico de los SSA, deben contemplar el uso de materiales encalantes.

El incremento observado en los niveles de $\mathrm{Ca}$ en el SSA, coincide con lo reportado por Combatt et al. (2007), quienes atribuyen este hecho a la solubilidad de la cal aplicada, lo cual probablemente permitió el suministro de iones calcio al suelo y, a su vez, contribuyó con el origen de $\mathrm{OH}^{-}$que reducen la acidez; de igual forma, Sadzawka \& Campillo (1993) señalan que el carbonato de $\mathrm{Ca}$ se disuelve a medida que los iones hidróxido son removidos de la solución del suelo, lo cual produce iones $\mathrm{Ca}$ y bicarbonato. Resultados similares obtuvieron Pool, Trinidad, Etchevers, Pérez \& Martínez (2000) y Hernández \& Viteri (2006), quienes evidenciaron un incremento significativo en Ca con la aplicación de cal dolomítica (Osorno, 2012).

Asimismo, el aumento registrado en la concentración de Mg en el SSA, concuerda con lo reportado por Pool et al. (2000), Osorno (2012), Acevedo (2007) y Combatt, Mercado \& Palencia (2009), quienes observaron un incremento significativo de este elemento con la aplicación de cal dolomita. Al respecto, Acevedo (2007) manifiesta que cuando el $\mathrm{Al}^{+3}$ se disuelve en un $\mathrm{pH}$ bajo, el suelo tiene una capacidad menor de retener $\mathrm{Ca}^{2+}$ y $\mathrm{Mg}^{2+}$, de manera que los iones de estos nutrientes tienen una mayor tendencia a lixiviarse del suelo; igualmente, Osorno (2012) y Lazcano (2001) indican que las enmiendas calcáreas se utilizan como equilibrantes de las bases, especialmente de Ca y Mg, y aumentan la disponibilidad de los mismos en el suelo.

El aumento leve de $\mathrm{K}$ que se presentó en el SSA, de acuerdo con Kamprath (1967), se debe a que el encalado al neutralizar el $\mathrm{Al}^{+3}$, Fe y el Mn intercambiables, aumenta los contenidos de las bases e incrementa su porcentaje de saturación. De igual forma, Zetina, Trinidad, Opereza, Volke \& Landois (2005) encontraron que el suelo encalado con cal dolomita mostró un mayor contenido de $\mathrm{Ca}, \mathrm{Mg}$ y $\mathrm{K}$ intercambiables; resultados similares obtuvieron Hernández \& Viteri (2006), quienes evidenciaron un aumento en la disponibilidad de este elemento con la aplicación de cal en SSA. El incremento en la concentración de $\mathrm{Na}$ por su parte, concuerda con lo reportado por Combatt et al. (2007), quienes afirman que este elemento presentó niveles mayores a los iniciales después del encalamiento, como consecuencia del desplazamiento de los minerales primarios de la fase intercambiable, con respecto a este catión. 
En cuanto a la respuesta de las plantas, el área foliar de la remolacha (B. vulgaris) que se sembró en el SSA disminuyó considerablemente en comparación con las plantas del suelo no sulfatado, esto indica que la disponibilidad de nutrientes en el suelo no sulfatado favoreció la expansión de las hojas y, por lo tanto, influyó en el aumento de esta variable, ya que de acuerdo con Plata, Forero, Balaguera \& Serrano (2009), la obtención de un buen nivel de área foliar se debe el aporte de nutrientes por parte del suelo. Asimismo, con respecto a la altura de las plantas, se podría inferir que la remolacha (B. vulgaris) redujo su crecimiento notablemente por efecto del tipo de suelo, lo cual se debe posiblemente a las condiciones particulares de los SSA por deficiencias o excesos de minerales básicos para su crecimiento, pues cuando se presentan deficiencias de nutrientes en el suelo, las plantas son muy pequeñas, tienen menor número de hojas y su crecimiento es mucho menor (Morales, 1995).

La respuesta positiva del nabo forrajero (B. rapa) en el SSA, puede explicarse probablemente a que esta especie se adapta fácilmente y tolera rangos de $\mathrm{pH}$ bajos (Young, 2012); igualmente, Birbaumer et al. (2000) manifiestan que el crecimiento del nabo forrajero ( $B$. rapa) favorece el reciclaje de nutrientes, especialmente fósforo y nitrógeno, además afirman que ésta planta se desarrolla satisfactoriamente aún en condiciones de lluvia escasa. De igual forma, Lazcano (2001) señala que ciertos cultivos son más tolerantes a la acidez del suelo que otros, y que la cal aporta el Ca necesario para el crecimiento y desarrollo de los mismos.

Complementariamente al incremento del contenido de Ca en el suelo tras la adición de enmiendas calcáreas, se produce también un aumento de $\mathrm{pH}$, lo cual permite una estabilización de contaminantes del suelo y una reducción de su biodisponibilidad y su absorción por las plantas (Knox, Seaman, Adriano, Pierzynski, 2000). Al respecto Taylor et al. (2000) y Watanabe \& Osaki (2002) indican que muchas especies vegetales tienen mecanismos eficientes para la exudación de iones a través de ácidos orgánicos por las raíces; de igual forma, la proliferación de determinadas rizobacterias y micorrizas estimulan el crecimiento de las plantas bajo condiciones ambientales estresantes, ya que sintetizan compuestos que facilitan la absorción de nutrientes como $\mathrm{N}$ y $\mathrm{P}$, además disminuyen o previenen los efectos negativos de los organismos fitopatógenos (Duñabeitia, Rodríguez, Salcedo \& Sarrionandia, 2004; Meharg \& Cairney, 2000; Leyval, Turnau \& Haselwandter, 1997).

Aunque el rábano forrajero ( $R$. sativus) presentó mayor crecimiento en el suelo no sulfatado, se obtuvo una respuesta positiva en el SSA. Los valores registrados en los dos tipos de suelo se encuentran dentro de los valores reportados por Nasevilla (2010) y Vibrans (2009) en condiciones normales de suelo; al respecto, Lazcano (2001) menciona que el encalado promueve el crecimiento de los cultivos e incrementa la absorción de agua y nutrimentos. Igualmente, Osorno (2012) manifiesta que esta técnica mejora las propiedades del suelo e incluso mejoras en la productividad de las plantas, de acuerdo con sus necesidades en elementos nutritivos y condiciones de $\mathrm{pH}$.

\section{Conclusiones}

Los resultados indicaron que la siembra de nabo forrajero (B. rapa) y rábano forrajero ( $R$. sativus), junto con el uso complementario de enmiendas calcáreas como correctivo, indujo un aumento en las concentraciones de $\mathrm{Ca}, \mathrm{Mg}, \mathrm{K}$ y $\mathrm{Na}$ en el suelo sulfatado ácido en invernadero y, a su vez, presentaron un mejor potencial de crecimiento y resistencia a los mismos; por ende, con la implementación de esta técnica se podría generar un ambiente propicio para aumentar su productividad.

Aunque la siembra de remolacha forrajera (B. vulgaris) en el SSA generó efectos positivos en la concentración de los nutrientes del suelo, su altura y área foliar presentó los valores más bajos como consecuencia de las características del suelo, lo cual limita su productividad. Sin embargo, se 
recomienda la validación de este estudio en campo para corroborar la respuesta de las especies como cultivos de cobertura o forraje, debido a que su implementación a nivel de invernadero no asegura el mismo comportamiento, por las diferentes condiciones climáticas u otros factores de alteración que puedan presentarse.

\section{Literatura citada}

1. Acevedo, O. (2007). Aluminio, un indicador de calidad ambiental en suelos de carga variable. Doctorado en Química, Universidad Autónoma del Estado de Hidalgo, México.

2. Bautista, Z., Delfín, G. \& Palacio J. (2011). Técnicas de muestreo para manejadores de recursos naturales. 2 ed. México D.C.: Centro de Investigaciones en Geografía Ambiental, Instituto de Geografía UNAM.

3. Birbaumer, G. \& Grupos temáticos del proyecto. (2000). Cultivar sin arar. Labranza mínima y siembra directa. Bogotá, Colombia: Proyecto de Conservación de Suelo y Agua en la Zona Andina - Proyecto Checua.

4. Cabrales, E. (2007). Dinámica nutrimental y caracterización microbiológica de los suelos sulfatados ácidos del valle del Sinú - Colombia. (Grupo de Investigación Manejo de cultivos tropicales) Córdoba, Colombia: Universidad de Córdoba.

5. Casierra, F. \& Aguilar O. (2007). Estrés por aluminio en plantas: reacciones en el suelo, síntomas en vegetales y posibilidades de corrección. Una revisión, Revista Colombiana de Ciencias Hortícolas, 1(2), 246-257.

6. Castro, H. \& Munevar, O. (2011). Mejoramiento químico integral de suelos ácidos mediante el uso combinado de materiales encalantes. En Sociedad venezolana de la Ciencia del Suelo. XIX Congreso Venezolano de la Ciencia del Suelo, Calabozo, Venezuela.

7. Combatt, E., Mercado, T. \& Palencia, G. (2009). Alteración Química de la solución de un suelo sulfatado acido, con encalamiento y lavado en columna disturbadas. Revista U.D.C.A. Actualidad \& Divulgación Científica, 12(1), $101-111$.

8. Combatt, E., Jarma, A., Martínez, G., Cabrales, E., Cardona, C., Atencio, L., Cadavid, C., Uparela, E. \& Mercado, J. (2007). Caracterización de suelos sulfatados ácidos y la respuesta de tres especies vegetales en el bajo Sinú de Córdoba. (Informe del Grupo de Investigación en Cultivos Tropicales de Clima Cálido, Oficina administrativa de Investigación y Extensión). Córdoba, Colombia: Universidad de Córdoba.

9. Combatt, E., Martínez, Z., Cabrales E., Martínez G., Castillo, C. \& Palencia M. (2005). Caracterización fisicoquímica y mineralógica de los suelos sulfatados ácidos en el transecto San Carlos - Cotorra- CarriIIo. (Grupo de Investigación en Cultivos Tropicales de Clima Cálido). Córdoba, Colombia: Universidad de Córdoba.
10. Combatt, E., Palencia, G. \& Marín, N. (2003). Clasificación de suelos sulfatados ácidos según azufre extraíble en los municipios del medio y bajo Sinú en Córdoba. Temas Agrarios, 8(2), 22-29.

11. Dakora, F. \& Phillips, D. (2002). Root exudates as mediators of mineral acquisition in low-nutrient environments. Plant and Soil, 245(1), 35-47.

12. Dent, D. \& Dawson, B. (2000). The acid test. An expert system for acid sulphate soils. This manual is intended to accompany the Evaluation edition (version 0.9) of the Acid Sulphate Soils Identikit.

13. Duñabeitia, M., Rodríguez, N., Salcedo, I. \& Sarrionandia, E. (2004). Field mycorrhization and its influence on the establishment and development of the seedlings in a broadleaf plantation in the Basque Country. Forest Ecology and Management, 195, 129-139.

14. Hernández, D. \& Viteri, S. (2006). Selección de abonos verdes para el manejo y rehabilitación de los suelos sulfatados ácidos de Boyacá (Colombia). Agronomía Colombiana, 24(1), 131-137.

15. Hinsinger, P., Plassard, C., Tang, C. \& Jaillard, B. (2003). Origins of root-mediated $\mathrm{pH}$ changes in the rhizosphere and their responses to environmental constraints: a review. Plant and Soil, 248(1-2), 43-59.

16. IGAC - Instituto Geográfico Agustín Codazzi. (2006). Métodos analíticos de laboratorio de suelos. 6 ed. Bogotá, Colombia: Imprenta Nacional.

17. Kamprath, E. (1967). Acidez del suelo y su respuesta al encalado. North Carolina, International Soil Testing. Raleigh, $15-24$.

18. Knox, A., Seaman, J., Adriano, D. \& Pierzynski, G. (2000). Chemophytostabilization of metals in contaminated soils. En: Wise, D. Trantolo, D., Cichon, E., Inyang, H. \& Stottmeister, U., (Eds.), Bioremediation of contaminated soils (pp. 811-836). New York: Marcel Dekker, Inc.

19. Lazcano, I. (2001). Cal agrícola: conceptos básicos para la producción de cultivos. International Plant Nutrition Institute - IPNI. Recuperado de http://www.ipni. net/ppiweb/iamex.nsf/\$webindex/5057DEAFC8DE54C C06256AD1005D7CB9/\$file/cal+agricola+conceptos+ basicos+para+la+produccion+de+cultivos.pdf

20. Leyval, C., Turnau, K. \& Haselwandter, K. (1997). Effect of heavy metal pollution on mycorrhizal colonization and function: physiological, ecological and applied aspects. Mycorrhiza, 7, 139 - 153.

21. Meharg, A. \& Cairney, J. (2000). Ectomycorrhizas extending the capabilities of rhizosphere remediation? Soil Biology \& Biochemistry, 32, 1475-1484.

22. Molina, E. Acidez de suelo y encalado (2008). Tomado de Molina, E. (1998) Encalado para la corrección de la acidez del suelo. ACCS. San José, Costa Rica. Recuperado de http://anfacal.org/media/Biblioteca_Digital/Agricultura/Neutralizacion_de_Suelos_Acidos/ JM-encalado_y_acidez.pdf

23. Montaño, J. \& Forero, F. (2013). Efecto de subproductos orgánicos del proceso panelero sobre propiedades físicas de un suelo sulfatado ácido. Corpoica Cienc. Tecnol. Agropecu., 14(2), 207 - 214. 
24. Morales, J. (1995). Cultivo de remolacha. Fundación de Desarrollo Agropecuario, Inc. Serie Cultivos, Boletín Técnico 22.

25. Nasevilla, J. (2010). Estudio de las características físicoquímicas y nutricionales de dos ecotipos de rábano (Raphanus sativus L). Ingeniería en Industrialización de Alimentos, Universidad Tecnológica Equinoccial, Quito, Ecuador.

26. National Working Party on Acid Sulfate Soils. (2000). National strategy for the management of coastal acid sulfate soils. Wollongbar, Australia: NSW Agriculture Wollongbar Agricultural Institute Bruxner Highway.

27. Ocampo, J., Chavarriaga, W. \& Ceballos, N. (2007). Valoración de tres fuentes de calcio en suelos ácidos de la granja tesorito y la respuesta en producción para el cultivo de arveja. Agron., 15(2), 7-15.

28. Osorno, H. (2012). Mitos y realidades de las cales y enmiendas en Colombia. Trabajo de grado, Universidad nacional de Colombia, Medellín, Colombia.

29. Plata, A., Forero F., Balaguera, E. \& Serrano P. (2009). Evaluación del efecto de la aplicación de cachaza fresca al cultivo de repollo (Brassica oleraceae var. capitata L.). Ciencia y Agricultura, 7(1), 29 - 42.

30. Pool, L., Trinidad, A., Etchevers J., Pérez, J. \& Martínez, A. (2000). Mejoradores de la fertilidad del suelo en la agricultura de ladera de los altos de Chiapas, México. Agrociencia, 34, 251-259.
31. Sadzawka, A. \& Campillo, R. (1993). Problemática de la acidez de los suelos de la IX región. IPA Carillanca, 12(3), 3 - 12.

32. Taylor, G., McDonald, J., Hunter, D., Bertsch, P., Elmore, D., Rengel, Z., Reid, R. (2000). Direct measurement of aluminum uptake and distribution in single cells of Chara corallina. Plant Physiol., 123, 987 - 996.

33. Vibrans, H. (Ed.). (2009). Ficha: Raphanus sativus $L$, Malezas de México. Recuperado de http://www.conabio.gob.mx/malezasdemexico/brassicaceae/raphanussativus/fichas/ficha.htm

34. Watanabe, T. \& Osaki, M. (2002). Mechanisms of adaptation to high aluminum condition in native plant species growing in acid soils: a review. Commun. Soil Sci. Plant anal., 33(7\&8), 1247 - 1260.

35. Young Mathews, A. (2012). Plant guide for field mustard (Brassica rapa ssp. rapa). USDA-Natural Resources Conservation Service, Plant Materials Center, CorvaIlis, OR. Published.

36. Zetina, R., Trinidad, A., Opereza, J., Volke, V. \& Landois, L. (2005). Relación bases intercambiables - rendimiento de maíz en un cambisol dístrico con labranza, encalado y abono verde. Terra Latinoamericana, 23(3), $389-397$ 